\title{
Study on influence factors of underwater shockwave sensor dynamic calibration
}

\author{
Hongtao Xü ${ }^{1}$, Penggang $\mathrm{Jin}^{2}$, Zan Gao ${ }^{3}$ \\ Xi'an Modern Chemistry Research Institute, Xi'an, 710065, China \\ ${ }^{2}$ Corresponding author \\ E-mail: ${ }^{1}$ baboon007@163.com, ${ }^{2}$ pluspeng@yeah.net, ${ }^{3}$ gaozan2003@163.com
}

Received 19 December 2019; accepted 28 December 2019 DOI https://doi.org/10.21595/vp.2019.21248

Check for updates

Copyright (C) 2020 Hongtao Xu, et al. This is an open access article distributed under the Creative Commons Attribution License, which permits unrestricted use, distribution, and reproduction in any medium, provided the original work is properly cited.

\begin{abstract}
To test coefficient sensitivity of underwater shockwave pressure sensor, dynamic calibration is conducted using traceable standard bench sensor and PCB913B02 sensor calibration equipment. In the research, silicon oil of different specification was filled into sealed tank in order to study the relations between silicon oil specification and calibration result of pressure sensor. Explosion tests are conducted using explosives of different formulas and underwater shockwave sensors filled with silicon oils of different specifications. The test result shows such principle and the method of calibration mentioned above could ensure the calibration precision, which therefore certifies the accuracy of shockwave pressure test.
\end{abstract}

Keywords: silicon specification, underwater pressure sensor, coefficient sensitivity.

\section{Introduction}

In the 8th session of the International Study Group for the Standardization of the Methods of Testing Explosives (EXTEST), the participants discussed the underwater explosion measurement and test method for determining the function of explosives. The test sensors used in various countries are mainly the ICP type of tourmaline underwater sensors produced by American PCB Company [1].

The sensitivity coefficient of such a sensor changes after repeated use, which greatly affects the reliability of the test results. China's Zhang Li [2] conducted research in this aspect, using TNT drug pack for dynamic calibration, the calibration is based on the classical pressure calculation formula on underwater TNT explosive shock wave summed up by Cole [3]. It is even convenient to use a traceable dynamic calibration system. However, the sensor needs to be disassembled during sensor calibration. The sensor's built-in silicone oil is inevitably lost in this process. This requires us to find a viable alternative silicone oil to meet the calibration and use requirements of the sensors.

\section{Test part}

\subsection{Instruments and samples}

The schematic diagram of the experimental system is shown in Fig. 1. The test instrument is the PCB913B02 sensor calibration device, and we choose PCB136A as the standard sensor, and using Multipro data acquisition system produced by Nichols. 5 kinds of silicone oil, the specifications are shown in Table 1.

\subsection{Experimental principle}

In order to make the test effect more obvious, we selected two new PCB138 7525 and PCB138 7521 sensors. Fill multiple cylinders with different specifications of silicone oil. Install the above two sensors that have been disassembled into exposed tourmaline. The sensor and silicone oil are shown in Fig. 2. 
Table 1. The specification of silicone oil

\begin{tabular}{|c|c|c|}
\hline Name and lot number & Density $\left(\rho / \mathrm{g} \cdot \mathrm{cm}^{-3}\right)$ & Kinematic viscosity $\left(v 20^{\circ} \mathrm{C} / \mathrm{mm}^{2} \mathrm{~s}^{-1}\right)$ \\
\hline PCB sensor with silicone oil & 0.9458 & 19.9 \\
\hline First batch of samples & 0.9390 & 15 \\
\hline The second batch of samples (1\#) & 0.9450 & 21.16 \\
\hline The second batch of samples (2\#) & 0.9450 & 21.39 \\
\hline Home-made sensor with silicone oil & 0.9710 & 345.5 \\
\hline
\end{tabular}

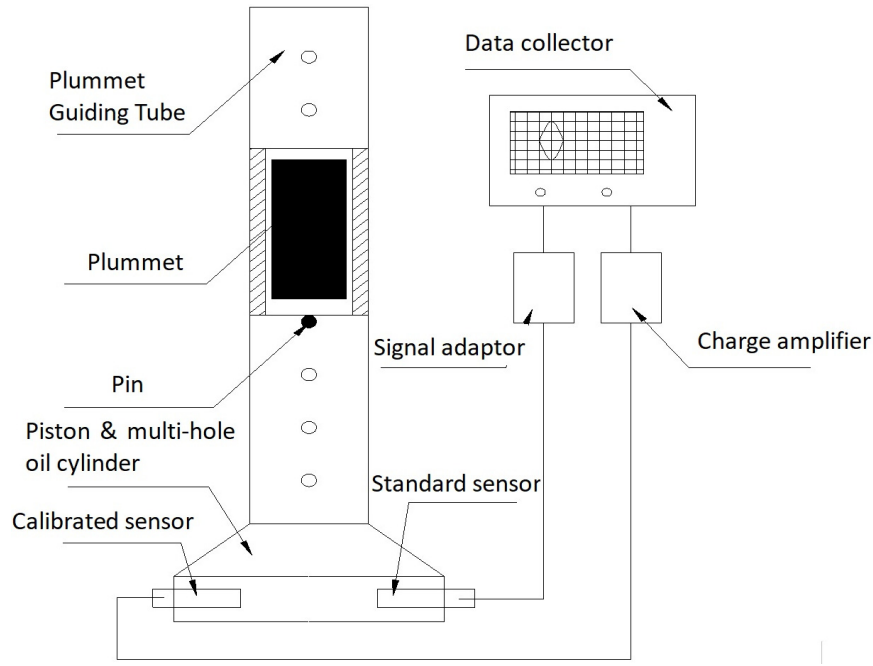

Fig. 1. PCB913B02 hydraulic pulse generator
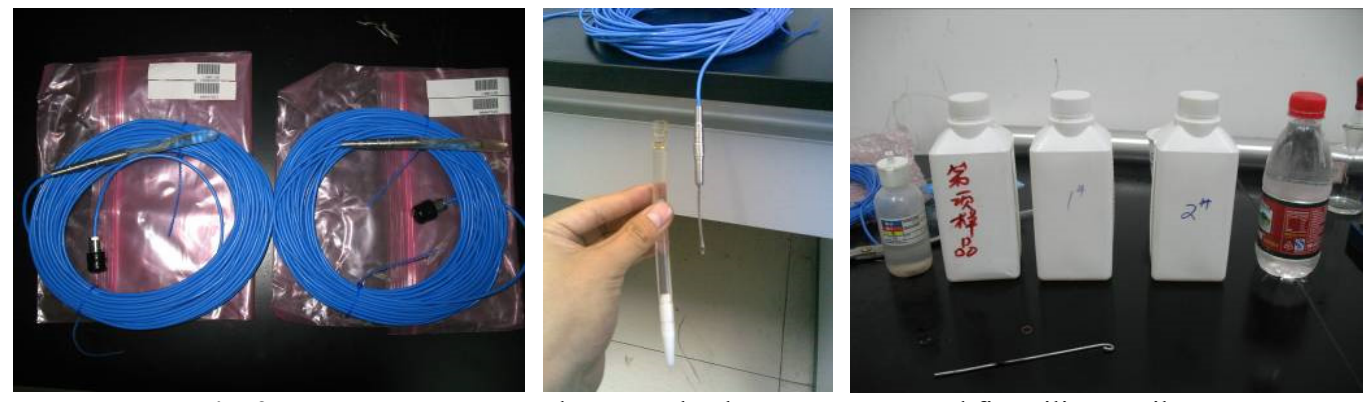

Fig. 2. Test two new ICP underwater shock wave sensors and five silicone oils

The principle of the calibration device is to use a mass block falling from a certain height to hit a piston filled with a silicone oil-tight pressure cylinder to generate a pressure pulse with a pulse width of $7 \mathrm{~ms}-8 \mathrm{~ms}$. The generated calibration pressure waveform is shown in Fig. 3. Since the internal pressure of the closed liquid is all equal everywhere, so the pressure pulse of this amplitude acts on all over the cylinder wall. The cylinder wall with pressure is equipped with a PCB136A reference sensor as the pressure transmission standard, which is traced back to NIST. The calibrated sensor is mounted at the symmetrical position of the cylinder wall with pressure and the sensitivity is dynamically calibrated by using the comparison method.

The $136 \mathrm{~A}$ pressure transmission standard is to suspend a single piece of tourmaline piezoelectric crystal between two steel rods, its diameter is $3.8 \mathrm{~mm}$ and thickness is $0.127 \mathrm{~mm}$. Since no diaphragm and outer casing affect the inherent characteristics of tourmaline crystal, therefore, the precision is high. Its shape is shown in Fig. 4. 


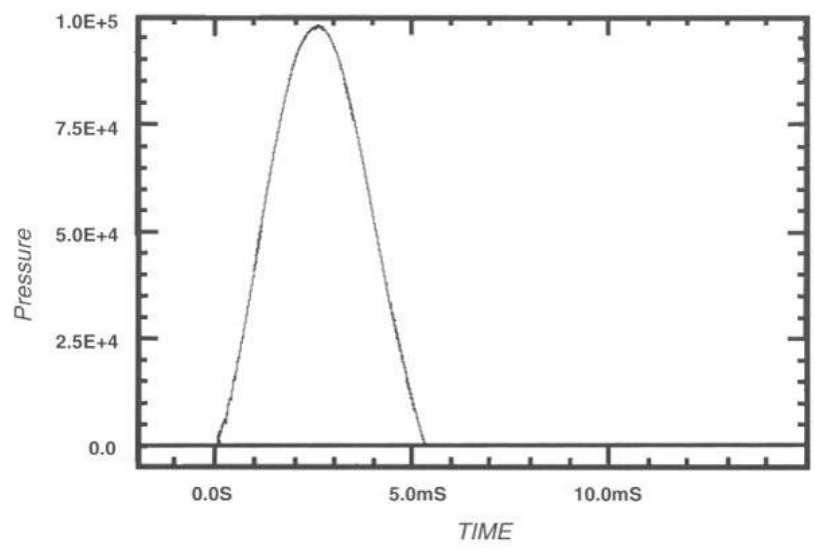

Fig. 3. Typical pressure pulse signal generated by model 913B02

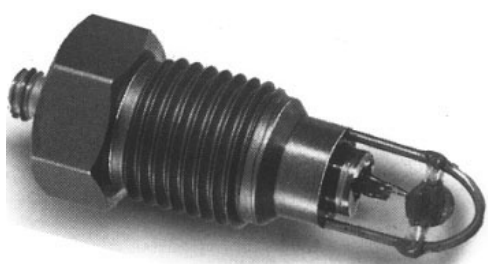

Fig. 4. 136A standard sensor

\section{Results and discussion}

\subsection{Analysis of sensor sensitivity coefficient under the calibrations of different silicone oils}

In the factory's certificate of a sensor, there is a description on the traceable source of the zero-calibrated sensor and its technical indicators. The dynamic calibration device used is the PCB913B02 sensor calibration device, and the test method used is AT601-8 (NIST). The technical indicators are mainly sensitivity and linearity. There is no relevant standard in China. According to the sensor factory's certificate and the instructions of the calibration device, the calibration process is carried out at a temperature of $23{ }^{\circ} \mathrm{C}$ and a relative humidity of $43 \%$. Using the different oils, the sensor is calibrated at 5 points in the measuring range, and the obtained data is straightline fitted by the least squares method so as to obtain the sensitivity coefficient.

The dynamic calibration sensitivity coefficient of the two sensors under different silicone oil systems is shown in Table 2.

Table 2. Dynamic calibration sensor sensitivity coefficient under different kinds of silicone oil systems

\begin{tabular}{|c|c|c|}
\hline Batch & 7525 sensor & 7521 sensor \\
\hline Factory's certificate & $142.7 \mathrm{mv} / \mathrm{MPa}$ & $141.6 \mathrm{mv} / \mathrm{MPa}$ \\
\hline Sensor's original-filled oil & $141.82 \mathrm{mv} / \mathrm{MPa}$ & $140.85 \mathrm{mv} / \mathrm{MPa}$ \\
\hline 1st batch & $139 \mathrm{mv} / \mathrm{MPa}$ & $139.98 \mathrm{mv} / \mathrm{MPa}$ \\
\hline 2nd batch 1\# & $139.13 \mathrm{mv} / \mathrm{MPa}$ & $140.11 \mathrm{mv} / \mathrm{MPa}$ \\
\hline 2nd batch 2\# & $140.82 \mathrm{mv} / \mathrm{MPa}$ & $140.28 \mathrm{mv} / \mathrm{MPa}$ \\
\hline Home-made sensor oil & $144.04 \mathrm{mv} / \mathrm{MPa}$ & $143.75 \mathrm{mv} / \mathrm{MPa}$ \\
\hline
\end{tabular}

In the test conditions of five different silicone oil sealed cylinders, the sensitivity coefficients of the two sensors did not change greatly. The sensitivity of the dynamic calibration of the silicone oil contained in the sensor is the closest to that of the factory's certificate. The maximum deviation between the sensitivity coefficient value and the factory certificate's value is $1 \%$. 


\subsection{Linearity analysis of the sensor sensitivity coefficient under different silicone oil calibrations}

The sensitivity coefficient of the sensor at each calibration point on its range during calibration is inconsistent. However, in order to measure which sensor's applicability is better, the linearity of its sensitivity coefficient is calculated by using the least squares method in the full-range segment. The data is shown in Table 3.

Table 3. Dynamically calibrate the sensor's sensitivity coefficient linearity under different kinds of silicone oil systems

\begin{tabular}{|c|c|c|}
\hline Batch & 7525 sensor & 7521 sensor \\
\hline Factory's certificate & $0.4 \% \mathrm{FS}$ & $0.9 \% \mathrm{FS}$ \\
\hline Sensor's original-filled oil & $0.26 \% \mathrm{FS}$ & $0.49 \% \mathrm{FS}$ \\
\hline 1st batch & $0.36 \% \mathrm{FS}$ & $0.43 \% \mathrm{FS}$ \\
\hline 2nd batch 1\# & $0.63 \% \mathrm{FS}$ & $1.39 \% \mathrm{FS}$ \\
\hline 2nd batch 2\# & $1.04 \% \mathrm{FS}$ & $1.97 \% \mathrm{FS}$ \\
\hline Home-made sensor oil & $1.5 \% \mathrm{FS}$ & $1.28 \% \mathrm{FS}$ \\
\hline
\end{tabular}

The linearity of the sensitivity coefficient of the sensor is a characteristic of the sensitive component itself, but the linearity of its sensitivity coefficient changes with the type of silicone oil filled in it during the test.

Test the underwater explosion energy for sensors filled with different silicone oils for applicable comparison.

The test samples are mainly RDX \& HMX-based 18 formulas containing AL and B and about $30 \mathrm{~g}$ of press-fit explosives with $8 \#$ detonator hole. In order to ensure the stability of the test, this test is to ignite two sensors and the third one is a backup. Using 3 sensors, it is 7525, 7521 and 7500. Among them, the 7525 sensor is filled with the second batch of $1 \#$ silicone oil, the 7521 sensor is filled with silicone oil used for home-made sensors, and the 7500 is an undissembled sensor. In the test process the three sensors were bundled together. It is judged whether the distance from the sensors to the explosion centre is consistent, based on the arrival time of the shock wave. By calculating the test dose, the depth entered in the water, and the ranging distance has been determined, the test results have removed the boundary effect caused by the explosion. The test system uses Agilent VXI data acquisition instrument, as well as PCB482A05 signal adapter. Data processing is performed by using self-developed data processing software. The test site is shown in Fig. 5, which is a pool, a lifting sensor, and an electrical measuring system.
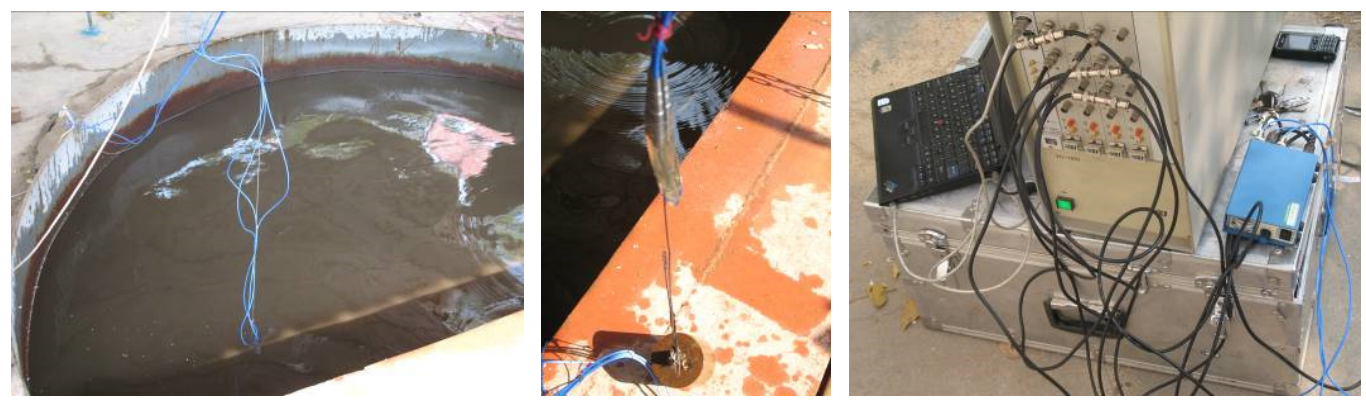

Fig. 5. Pool, lifting sensor, electrical measuring system

The 18 kinds of different formula explosives were sorted out, and the test data obtained from the underwater explosion was used for the test results. The pressure corresponding to each sensor was shown in Fig. 6.

When the source of the explosion explodes in the water, the shock wave is transmitted from the aqueous medium to the sensor's silicone oil, then to the sensor's sensitive component 
tourmaline. In this process, maybe there exists an impedance matching problem among the acoustic impedance of water and the acoustic impedance of silicone oil and the acoustic impedance of the sensor casing. The acoustic characteristics of the materials above did not change during the calibration process of the sensor, among which only the kinematic viscosity change of the silicone oil is involved. In the process of the shock wave propagating in the silicone oil, its impact process is very short, and the silicone oil sealed in the sensor cavity is not deformed and is relatively stationary. Therefore, there is only compressive stress inside the silicone oil. From the Euler motion differential equation of the ideal fluid, the pressure transmitted to the sensor-sensitive component tourmaline will not change significantly. It is found in the comparison test of different explosives that after we changed the different motion viscosity of the silicone oil, the output of the sensor (the sensitivity coefficient is the dynamic calibration sensitivity coefficient for filling the silicone oil) under the same explosion source is changed as shown in Fig. 3. This explains that under the action of shock wave, we cannot ignore the viscosity of the liquid for the process of transmitting pressure in the sealing the silicone oil. i.e. there is not only stressing pressure but also shearing stress in this process [6]. At this time, the shearing stress mainly comes from the momentum exchange between the molecules inside the silicone oil. The silicone oils with different kinematic viscosities have different shearing stresses during this impact process, and the greater the kinematic viscosity, the greater the shearing stress. This also causes the pressure of testing the PCB underwater shock wave sensor with home-made silicone oil to be small. In the underwater explosion performance test of different formula of explosives, the deviation of testing shock wave pressure is up to $11 \%$.

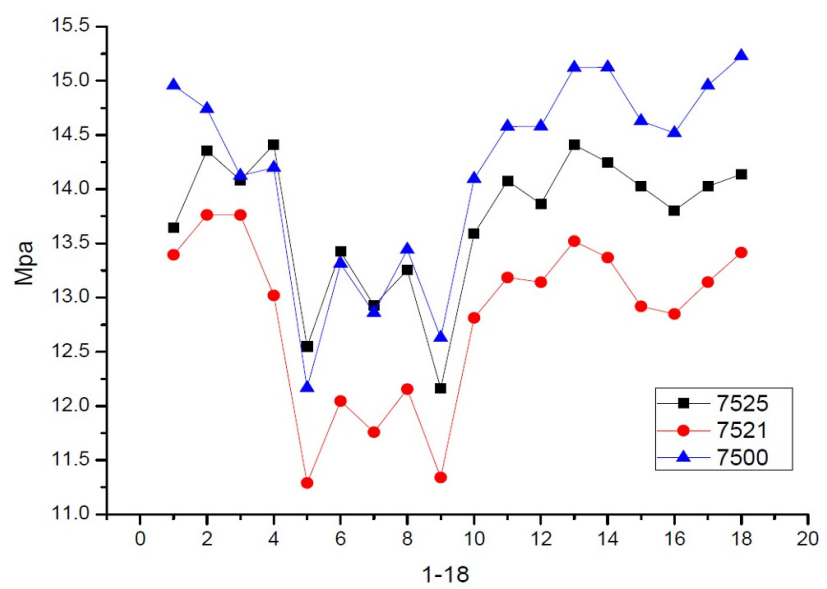

Fig. 6. Pressure results

\section{Conclusions}

1) We carried out the dynamic calibration of the sensitivity coefficient of the underwater shock wave sensor by using the PCB913B02 dynamic calibration device. The calibration principle and method are feasible.

2) The selection of the liquid medium in the sealed pressure cylinder of the calibration device affects the calibration result of the sensitivity coefficient, but has little effect on the linearity of the sensitivity coefficient.

3) As to the calibrating work or liquid medium filled in the PCB138 type of underwater shock sensor, if we use the silicone oil that is close to the density and kinematic viscosity in the original type of the PCB138 sensor, the deviation caused by filling different type of media in PCB138 sensor will be reduced in the course of calibrating and using it. 


\section{References}

[1] Persson J. P. EXTEST international study group for the Standardization of the Methods of Testing Explosives. Formerly European commission for the standardization of the tests of explosives. Propellants, Explosives, Pyrotechnics, Vol. 3, Issues 1-2, 1978, p. 23-28.

[2] Li Zhang Dynamically calibrate ICP pressure sensor for underwater explosions. Coal Mine Explosion, Vol. 46, Issue 3, 1999, p. 7-9.

[3] Cour P., et al. Underwater Explosion. National Defense Industry Press, 1960.

[4] Christmann W., Lingens P. J. P. Contribution to the standardization of the energy measurement of underwater explosives. Explosives, Vol. 5, 1980, p. 59-61.

[5] Bjarnholt G. Recommendations on underwater explosion measurement method standards and data calculation standards. Proceedings of the 8th Session of the International Research Institute for Standardization of Explosive Testing Methods, 1984.

[6] Zhang Yuelin, Chen Yunsheng Fluid Mechanics. Nanjing University of Science and Technology, 2017. 